\title{
Rac1 regulates platelet shedding of CD40L in abdominal sepsis
}

\author{
Rundk Hwaiz ${ }^{1}$ Milladur Rahman', Enming Zhang ${ }^{2}$ and Henrik Thorlacius ${ }^{1}$
}

Matrix metalloproteinase-9 (MMP-9) regulates platelet shedding of CD40L in abdominal sepsis. However, the signaling mechanisms controlling sepsis-induced shedding of CD40L from activated platelets remain elusive. Rac1 has been reported to regulate diverse functions in platelets; we hypothesized herein that Rac1 might regulate platelet shedding of CD40L in sepsis. The specific Rac1 inhibitor NSC23766 (N6-[2-[[4-(diethylamino)-1-methylbutyl] amino]-6methyl-4-pyrimidinyl]-2 methyl-4, 6-quinolinediamine trihydrochloride) was administered to mice undergoing cecal ligation and puncture (CLP). Levels of CD40L and MMP-9 in plasma, platelets, and neutrophils were determined by use of ELISA, western blot, and confocal microscopy. Platelet depletion abolished the CLP-induced increase in plasma levels of CD40L. Rac1 activity was significantly increased in platelets from septic animals. Administration of NSC23766 abolished the CLP-induced enhancement of soluble CD40L levels in the plasma. Moreover, Rac1 inhibition completely inhibited proteinase-activated receptor-4-induced surface mobilization and secretion of CD40L in isolated platelets. CLP significantly increased plasma levels of MMP-9 and Rac1 activity in neutrophils. Treatment with NSC23766 markedly attenuated MMP-9 levels in the plasma from septic mice. In addition, Rac1 inhibition abolished chemokine-induced secretion of MMP-9 from isolated neutrophils. Finally, platelet shedding of CD40L was significantly reduced in response to stimulation with supernatants from activated MMP-9-deficient neutrophils compared with supernatants from wild-type neutrophils, indicating a direct role of neutrophil-derived MMP-9 in regulating platelet shedding of CD40L. Our novel data suggest that sepsis-induced platelet shedding of CD40L is dependent on Rac1 signaling. Rac1 controls surface mobilization of CD40L on activated platelets and MMP-9 secretion from neutrophils. Thus, our findings indicate that targeting Rac1 signaling might be a useful way to control pathologic elevations of CD40L in the systemic circulation in abdominal sepsis.

Laboratory Investigation (2014) 94, 1054-1063; doi:10.1038/labinvest.2014.92; published online 21 July 2014

Management of patients with sepsis poses a major challenge to clinicians and is largely limited to supportive therapies. In fact, sepsis is still a significant cause of mortality in intensive care units, which is related to an incomplete knowledge about the pathophysiology in sepsis. ${ }^{1-3}$ Intestinal perforation contaminates the abdominal cavity with toxins and microbes, evoking local formation of proinflammatory compounds, which can leak into the circulation causing a systemic inflammatory response. ${ }^{4,5}$ The lung is a sensitive and central target organ in polymicrobial sepsis. It is widely held that neutrophil infiltration is a rate-limiting step in septic lung damage. For example, inhibition of pulmonary accumulation of neutrophils by targeting specific adhesion molecules, such as CD11a, CD44, and CD162, has been shown to protect against septic lung injury. ${ }^{4,6,7}$ Accumulating studies have shown that platelets also have an important role in regulating pulmonary recruitment of neutrophils in abdominal sepsis. ${ }^{89}$ For example, it has been reported that platelet-derived CD40L is a potent inducer of neutrophil infiltration in septic lung injury. ${ }^{8}$ Soluble CD40L seems to induce increased plasma levels of CXC chemokines, which are potent stimulators of neutrophils. ${ }^{8}$ We recently showed that matrix metalloproteinase-9 (MMP-9) is an important regulator of CD40L shedding from platelets in abdominal sepsis. ${ }^{10}$ However, the detailed signaling mechanisms regulating sepsisinduced platelet secretion of CD40L are not known.

\footnotetext{
${ }^{1}$ Department of Clinical Sciences, Malmö, Section for Surgery, Lund University, Skåne University Hospital, Malmö, Sweden and ${ }^{2}$ Islet Pathophysiology, Lund University, Malmö, Sweden

Correspondence: Professor H Thorlacius, MD, PhD, Department of Clinical Sciences, Malmö, Section of Surgery, Lund University, Skåne University Hospital, 20502 Malmö, Sweden.

E-mail: henrik.thorlacius@med.lu.se

Received 11 March 2014; revised 20 May 2014; accepted 29 May 2014
} 
Extracellular stress situations, such as ischemia and infection, trigger intracellular signaling cascades converging on specific transcription factors regulating gene expression of inflammatory mediators. ${ }^{11,12}$ This signal transmission is largely regulated by intracellular kinases phosphorylating downstream targets. ${ }^{13}$ For example, small $(\sim 21 \mathrm{kDa})$ guanosine triphosphatases of the Ras-homologus (Rho) family, such as Rho A-C, Cdc42, and Rac1, are known to act as molecular switches regulating numerous important cellular functions. ${ }^{14-16}$ Rac1 is a ubiquitously expressed signal transducer regulating numerous processes related to inflammatory reactions, such as cell adhesion, chemotaxis, vascular permeability, and cytoskeletal reorganization. ${ }^{17,18}$ Racl has been shown to be expressed in platelets and recent studies have demonstrated that Racl is essential for lamellipodia formation, granule secretion, clot retraction, and phospholipase $\mathrm{C} \gamma 2$ activation in platelets. ${ }^{19-24}$ Moreover, targeting Racl signaling has been demonstrated to exert anti-inflammatory effects in models of reperfusion injury, endotoxemia, acute pancreatitis, and sepsis. ${ }^{17,25-27}$ Based on the findings that Racl regulates pleiotropic functions of platelets and that Racl appears to have an important role in diverse models of inflammation, we hypothesized in the present study that Racl might be involved in the regulation of platelet shedding of CD40L in abdominal sepsis.

\section{MATERIALS AND METHODS Animals}

Experiments were performed using male C57BL/6 mice weighing $20-25 \mathrm{~g}$. All experimental procedures were performed in accordance with the legislation on the protection of animals and were approved by the Regional Ethical Committee for Animal Experimentation at Lund University, Sweden. Animals were anesthetized by administration of $7.5 \mathrm{mg}$ (intraperitoneally) ketamine hydrochloride (Hoffman-La Roche, Basel, Switzerland) and $2.5 \mathrm{mg}$ (intraperitoneally xylazine (Janssen Pharmaceutica, Beerse, Belgium) per $100 \mathrm{~g}$ body weight.

\section{Experimental Model of Sepsis}

Polymicrobial sepsis was provoked by ligating and puncturing the cecum in mice as previously described in detail. ${ }^{28}$ Briefly, animals were anesthetized and the cecum was exteriorized and a ligature was placed below the ileocecal valve. The cecum was punctured two times with a 21-G needle. The cecum was then returned into the peritoneal cavity and the abdominal wall was closed with a suture. A platelet-depleting antibody directed against murine CD42b ( GP $1 \mathrm{~b} \alpha$, rat IgG, $1.0 \mathrm{mg} / \mathrm{kg}$; Emfret Analytics $\mathrm{GmbH} \mathrm{KG}$, Wurzburg, Germany) was given intraperitoneally $2 \mathrm{~h}$ before cecal ligation and puncture (CLP). A nonfunctional isotype control antibody (clone R3-34; BD Biosciences Pharmingen, San Jose, CA, USA) was administered intraperitoneally before CLP induction. To delineate the role of Racl inhibitor, animals were treated with vehicle $\left(\mathrm{dH}_{2} \mathrm{O}\right)$ or with $5 \mathrm{mg} / \mathrm{kg}$ of Rac1 inhibitor, NSC23766 (N6-[2-[[4-(diethylamino)1-methylbutyl] amino]-6-methyl-4-pyrimidinyl]-2 methyl-4, 6-quinolinediamine trihydrochloride; Tocris Bioscience, Bristol, UK), intraperitoneally $30 \mathrm{~min}$ before CLP induction. Sham mice underwent the same surgical procedures except ligation and puncture of the cecum. Animals were reanesthetized at indicated time points after CLP induction.

\section{ELISA}

Plasma and supernatant levels of soluble CD40L and plasma level of MMP-9 were assayed $6 \mathrm{~h}$ after induction of CLP by use of commercially available ELISA Kits (R\&D Systems) using recombinant murine soluble CD40L and MMP-9 as standards.

\section{Platelet Isolation and CD40L Shedding}

Blood was collected in 1-ml syringes containing $0.1 \mathrm{ml}$ of acid-citrate-dextrose anticoagulant, immediately diluted with equal volumes of modified Tyrode solution $(1 \mu \mathrm{g} / \mathrm{ml}$ prostaglandin $\mathrm{E}_{1}$ and $0.1 \mathrm{U} / \mathrm{ml}$ apyrase), and centrifuged at $200 \mathrm{~g}$ for $5 \mathrm{~min}$ at room temperature. Platelet-rich plasma was collected and centrifuged at $800 \mathrm{~g}$ for $15 \mathrm{~min}$ at room temperature, and pellets were resuspended in modified Tyrode solution. After being washed one more time at $10000 \mathrm{~g}$ for $5 \mathrm{~min}$, platelets were resuspended at a count of $0.5 \times 10^{8}$ platelets per tube in Tyrode solution. Platelets were stimulated with proteinase-activated receptor-4 (PAR4) $(200 \mu \mathrm{M})$ (thrombin receptor-activating peptide; Bachem, Weil am Rhein, Germany) at $37^{\circ} \mathrm{C}$ with and without NSC23766 (10 and $100 \mu \mathrm{M})$. After stimulation, cells were immediately fixed by the addition of $0.5 \%$ paraformaldehyde, samples were centrifuged at $10000 \mathrm{~g}$ for $10 \mathrm{~min}$ at $4{ }^{\circ} \mathrm{C}$, and the soluble CD40L that was released was measured in the supernatant by ELISA, according to the manufacturer's protocol. In separate experiment, platelets from sham and CLP mice were isolated and lysed for active Racl pull-down assay and western blot was performed to measure the GTP-Racl as described below. For platelet confocal microscopy, $0.5 \times 10^{6}$ isolated platelets were seeded onto a chamber slide coated with fibrinogen $(20 \mu \mathrm{g} / \mathrm{ml})$. Adherent platelets were stimulated with PAR4 $(200 \mu \mathrm{M})$ at $37^{\circ} \mathrm{C}$ with and without NSC23766 $(10 \mu \mathrm{M})$ and fixed with $2 \%$ paraformaldehyde for $10 \mathrm{~min}$, and then washed and blocked with $2 \%$ bovine serum albumin for $30 \mathrm{~min}$, followed by incubation with a rabbit polyclonal primary antibody against MMP-9 (Abcam, Cambridge, MA, USA) for $1 \mathrm{~h}$ at room temperature. Chamber slides were washed and incubated with fluorescein isothiocyanate (FITC)-conjugated anti-rabbit secondary antibody (Cell Signaling Technology, Beverly, MA, USA) and platelet-specific phycoerythrin (PE)conjugated anti-CD41 (clone MWReg30, integrin $\alpha$ IIb chain, and rat IgG1) for $1 \mathrm{~h}$. Chamber slides are washed three times and confocal microscopy was performed using Meta 510 confocal microscopy (Carl Zeiss, Germany) by a $\times 63$ oil immersion objective $(\mathrm{NA}=1.25)$. FITC and PE were excited 
by 488 and $543 \mathrm{~nm}$ laser lines, corresponding emission wavelengths of FITC and PE were collected by the filters of 500-530 and 560-590 nm, respectively. The pinhole was $\sim 1$ airy unit and the scanning frame was $512 \times 512$ pixels. The fluorescent intensity was calculated by ZEN2009 software.

\section{Neutrophil Isolation and MMP-9 Secretion}

Bone marrow neutrophils were freshly extracted from femurs and tibias of healthy mice by aseptically flushing the bone marrow with the complete culture medium RPMI-1640 and then subsequently isolated by using magnetic beads; neutrophils were isolated from bone marrow using Anti-Ly6G-Biotin and Anti-Biotin MicroBeads (Miltenyi Biotec) as per the manufacturer's instructions (neutrophils purity was over $93 \%$ as determined by flow cytometry). About $1 \times 10^{6}$ neutrophils were preincubated with NSC23766 $(10 \mu \mathrm{M})$ for $20 \mathrm{~min}$ before challenging with $0.3 \mu \mathrm{g} / \mathrm{ml}$ recombinant mouse CXCL2 (R\&D Systems) or PBS as a control for $30 \mathrm{~min}$ at $37^{\circ} \mathrm{C}$. Cells were lysed for pull-down assay and western blot was used to detect GTP-Racl in neutrophils as described below. For confocal microscopy, freshly isolated $0.5 \times 10^{6}$ neutrophils were resuspended in PBS-BSA-CaCl 2 buffer (PBS containing $0.2 \% \mathrm{BSA}$ and $0.5 \mathrm{mM} \mathrm{CaCl}_{2}$ ) and activated with mouse CXCL2 with and without NSC23766 $(10 \mu \mathrm{M})$ as described above. Neutrophils were fixed with $2 \%$ paraformaldehyde and washed with PBS-BSA-CaCl 2 followed by permeabilization with $\mathrm{PBS}-\mathrm{BSA}-\mathrm{CaCl}_{2}$ Saponin buffer (PBS-BSA-CaCl ${ }_{2}$ buffer containing $0.01 \%(\mathrm{w} / \mathrm{v})$ saponin) for $10 \mathrm{~min}$ on ice. After permeabilization, cells are incubated with rabbit polyclonal primary antibody against MMP-9 (Abcam) for $1 \mathrm{~h}$ on ice. Cells were washed and incubated with FITC-conjugated anti-rabbit secondary antibody (Cell Signaling Technology, Beverly, MA, USA) and PE-conjugated anti-Ly-6G antibody (clone 1A8, rat IgG2a; BD Pharmingen, San Jose, CA, USA), which selectively binds to neutrophils for $1 \mathrm{~h}$ on ice. After labeling, cells were washed three times with saponin buffer and allowed to settle down on chamber slides for confocal imaging as described above.

\section{Pull-Down Assay and Western Blotting}

Racl activity was determined by active Racl pull-down and detection kit using the protein binding domain of GSTPAK1, which binds with the GTP-bound form of Rac1 (Pierce Biotechnology, Rockford, IL, USA). Briefly, cells were resuspended in lysis buffer on ice, and then centrifuged at $16000 \mathrm{~g}$ for $15 \mathrm{~min} ; 10 \mu \mathrm{l}$ from each supernatant was removed to measure the protein content using Pierce BCA Protein Assay Reagent (Pierce Biotechnology) and the rest of the volume was used for the pull-down assay. Supernatant containing equal amount of proteins were then diluted with $2 \times$ SDS sample buffer and boiled for $5 \mathrm{~min}$. Proteins were separated using SDS-PAGE (10-12\% gel). After transferring to a nitrocellulose membrane (Bio-Rad, Hercules, CA, USA), blots were blocked with Tris-buffered saline/Tween-20 containing $3 \%$ bovine serum albumin at room temperature for $1 \mathrm{~h}$, followed by incubation with an anti-Racl antibody (1:1000) at $4{ }^{\circ} \mathrm{C}$ overnight. Binding of the antibody was detected using peroxidase-conjugated anti-mouse antibody (1:100 000; Pierce Biotechnology) at room temperature for $2 \mathrm{~h}$ and developed by Immun-Star WesternC Chemiluminescence Kit (Bio-Rad). Total Racl was used as a loading control. To measure the MMP-9 level in the plasma, $20 \mu \mathrm{g}$ of plasma was loaded onto an SDS-polyacrylamide gel for electrophoresis, and then transferred onto immunoblot membranes. The membranes were blocked with Tris-buffered saline/Tween-20 containing 5\% non-fat milk for $1 \mathrm{~h}$, and incubated with an anti-MMP-9 antibody (Abcam) at $4{ }^{\circ} \mathrm{C}$ over night. The membranes were then washed three times, and incubated with a horseradish peroxidase-coupled secondary antibody (Cell Signaling Technology) for $2 \mathrm{~h}$. Blots were again washed three times, and developed with the Immun-Star WesternC Chemiluminescence Kit (Bio-Rad). $\beta$-Actin was used as a loading control.

\section{Flow Cytometry}

For the analysis of platelet depletion, blood was collected into syringes containing 1:10 acid citrate dextrose at $6 \mathrm{~h}$ after CLP induction. Immediately after collection, blood samples were incubated with an anti-CD16/CD32 antibody for $10 \mathrm{~min}$ at room temperature to reduce non-specific binding of labeled antibody with Fcy III/II receptors. Neutrophils were labeled with PE-conjugated anti-Ly-6G and platelets were labeled with FITC-conjugated anti-CD41 (clone MWReg30, integrin $\alpha \mathrm{IIb}$ chain, and rat IgG1) antibodies and plotted as FL1 versus FL2 to determine the percentage of platelet depletion in the upper-right and lower-right area of the quadrant plot. For the purity of magnetically isolated neutrophils, we were first incubated with an anti-CD16/CD32 antibody for $10 \mathrm{~min}$ and then stained with PE-conjugated anti-Ly-6G and FITC-conjugated anti-Mac-1 (clone M1/70, integrin $\alpha \mathrm{M}$ chain, and rat IgG2b) antibodies. All antibodies, except where indicated, were purchased from eBioscience (San Diego, CA, USA). Flow cytometric analysis was performed according to standard settings on a FACScalibur flow cytometer (Becton-Dickinson, Mountain View, CA, USA) and a viable gate was used to exclude dead and fragmented cells.

\section{In vitro Shedding of Platelet-Derived CD40L}

Neutrophils from wild-type and MMP-9 gene-deficient mice were isolated as described above and a total of $1 \times 10^{6}$ wildtype and MMP-9 gene-deficient neutrophils per tube were incubated with $0.3 \mu \mathrm{g} / \mathrm{ml}$ recombinant mouse CXCL2 separately (R\&D Systems) for $30 \mathrm{~min}$ at $37^{\circ} \mathrm{C}$. After stimulation, samples were centrifuged at $10000 \mathrm{~g}$ for $5 \mathrm{~min}$ at $4{ }^{\circ} \mathrm{C}$ and supernatants were collected for further use. At the same time, platelets from MMP-9 gene-deficient mice were isolated as described above. Platelets were resuspended at a count of $0.5 \times 10^{8}$ platelets per tube in Tyrode solution and stimulated 
with $200 \mu \mathrm{M}$ of PAR4 and coincubated with equal volume of supernatants derived from wild-type or MMP-9 genedeficient neutrophils or with $\mathrm{PBS}$ at $37^{\circ} \mathrm{C}$ for $30 \mathrm{~min}$. After stimulation, cells were immediately fixed by adding $0.5 \%$ formaldehyde, where after samples were centrifuged at $10000 \mathrm{~g}$ for $10 \mathrm{~min}$ at $4{ }^{\circ} \mathrm{C}$, and soluble CD40L was measured in the supernatant by ELISA.

\section{Statistics}

Data were presented as mean values \pm s.e.m. Statistical evaluations were performed by using nonparametric test (Mann-Whitney). $P<0.05$ was considered significant and $n$ represents the total number of mice in each group. Statistical analysis was performed by using SigmaPlot 10.0 software (Systat Software, Chicago, IL, USA).

\section{RESULTS}

\section{Rac1 Activity in Platelets}

We first examined Rac1 activity (Rac1-GTP) in platelets. We observed that CLP increased Rac1-GTP levels in septic platelets compared with platelets from sham animals, showing that Racl is activated in platelets in septic animals (Figures 1a and b). Notably, administration of the Rac1 inhibitor NSC23766 abolished CLP-induced Racl activation in platelets (Figures 1a and b).

\section{Rac1 Regulates Platelet Shedding of CD40L}

CLP caused a 26-fold increase in the plasma levels of CD40L, that is, from $0.09 \pm 0.02$ to $2.40 \pm 0.30 \mathrm{ng} / \mathrm{ml}$ (Figure 2a). Administration of the anti-GP1b $\alpha$ antibody reduced systemic platelet counts by more than $85 \%$ in CLP animals (Figure $2 \mathrm{~b}$ ). Interestingly, we found that platelet depletion decreased plasma levels of CD $40 \mathrm{~L}$ by more than $95 \%$ in septic mice (Figure 2a). Administration of NSC23766 in control mice had no effect on plasma levels of soluble CD40L (Figure 2a). In contrast, we found that treatment with NSC23766 reduced soluble levels of CD40L in the plasma from septic mice from $2.40 \pm 0.30$ down to $0.145 \pm 0.02 \mathrm{ng} /$ $\mathrm{ml}$, corresponding to a $98 \%$ reduction (Figure 2a). To determine the direct role of Racl in regulating platelet expression and secretion of CD40L, isolated platelets were stimulated with PAR4 in vitro. It was found that PAR4 markedly increased surface expression of CD40L on platelets (Figures 2c and d). Notably, preincubation with NSC237666 significantly decreased PAR4-induced surface mobilization of CD40L on platelets (Figures $2 \mathrm{c}$ and $\mathrm{d}$ ). Moreover, we found that PAR4 caused substantial secretion of CD40L from platelets and that NSC23766 dose-dependently reduced PAR4-induced platelet secretion of CD40L (Figure 2e). For example, $100 \mu \mathrm{M}$ of NSC23766 decreased PAR4-induced platelet secretion of CD40L by $76 \%$ (Figure 2e).

\section{Rac1 Activity in Isolated Neutrophils}

Neutrophils were isolated from bone marrow as described in Materials and Methods section and Racl activity was
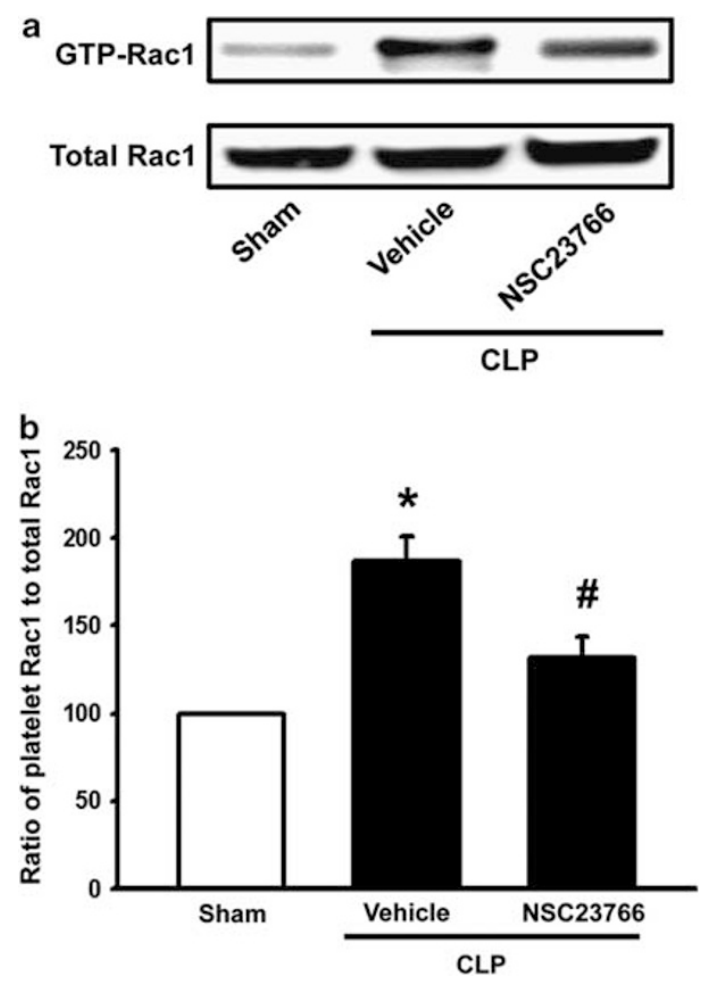

Figure 1 Rac1 activity in platelet lysate. (a) Rac1-GTP was determined by western blotting using GST-PAK pull-down beads $6 \mathrm{~h}$ after induction of cecal ligation and puncture (CLP). (b) Band intensities were quantified by densitometry and normalized to total Rac1. Western blots are representative of four independent experiments. Mice were treated with the Rac1 inhibitor NSC23766 (N6-[2-[[4-(diethylamino)-1-methylbutyl] amino]-6-methyl-4-pyrimidinyl]-2 methyl-4, 6-quinolinediamine trihydrochloride; $5 \mathrm{mg} / \mathrm{kg}$ ) or vehicle $\left(\mathrm{dH}_{2} \mathrm{O}\right) 30$ min before CLP induction. Sham-operated mice served as negative controls. Bars represent mean \pm s.e.m. and $n=4$. ${ }^{*} P<0.05$ vs sham and ${ }^{\#} P<0.05$ vs vehicle + CLP.

analyzed by western blotting. We found that CXCL2 stimulation markedly increased Racl activity in neutrophils and that preincubation with NSC23766 abolished the CXCL2-evoked activation of Rac1 in neutrophils (Figures 3a and b).

\section{Rac1 Regulates Neutrophil Secretion of MMP-9}

We have recently shown that MMP-9 regulates platelet shedding of $\mathrm{CD} 40 \mathrm{~L}^{10}$ and it was therefore of interest to examine the role of Rac1 in neutrophil secretion of MMP-9 in sepsis. Using ELISA, we found that CLP increased plasma levels of MMP-9 and the administration of NSC23766 decreased CLP-induced plasma levels of MMP-9 by 50\% (Figure 4a). Moreover, by use of western blot, it was found that MMP-9 expression in the plasma was significantly enhanced in septic animals (Figures 4b and c). Treatment with NSC23766 markedly reduced sepsis-provoked plasma expression of MMP-9 (Figures 4b and c). Knowing that neutrophils are a major source of MMP- $9,{ }^{29}$ we next analyzed MMP-9 


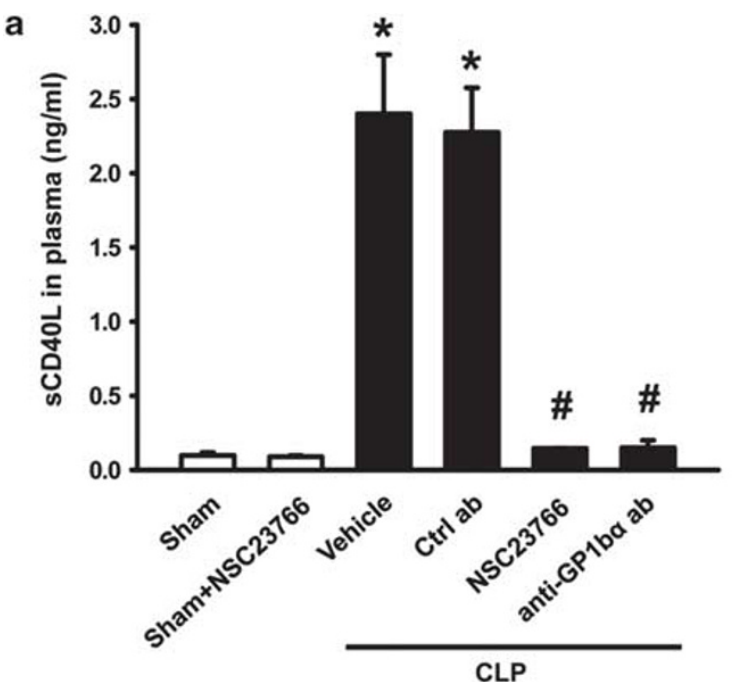

C
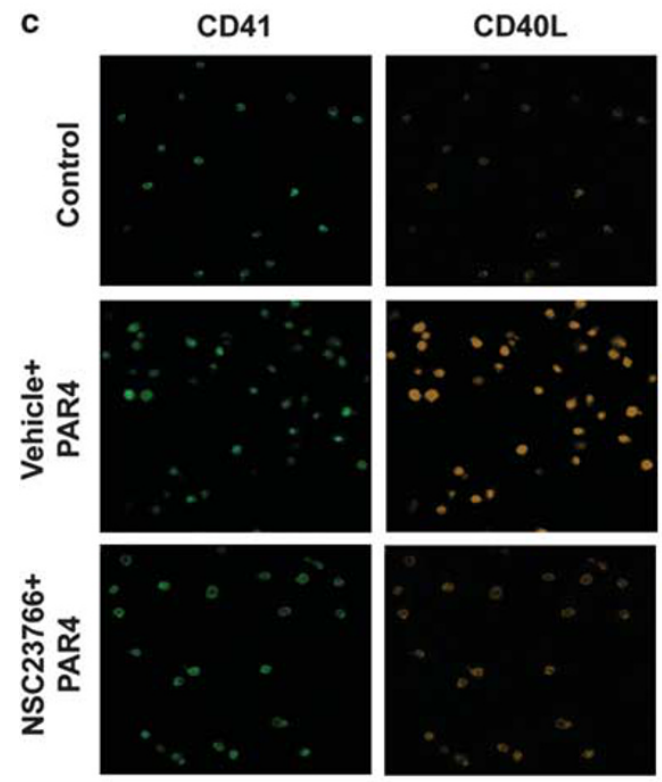

b
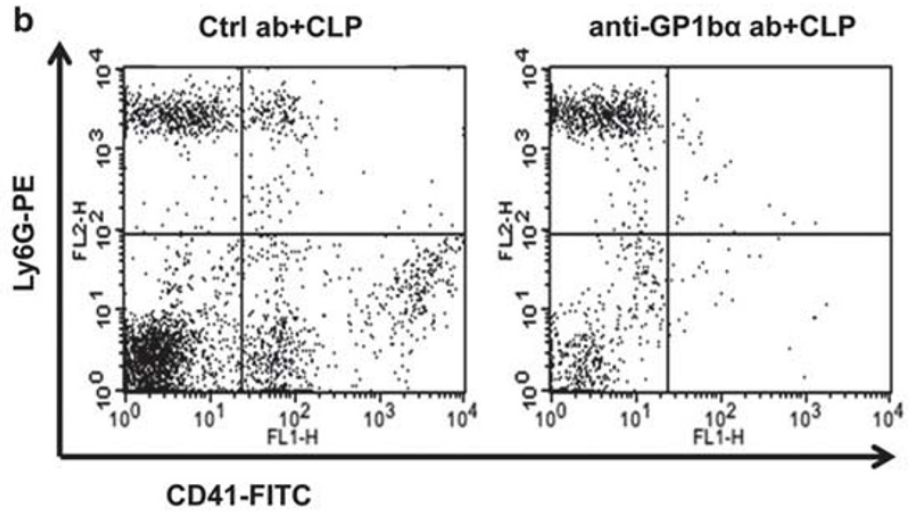

d

Merge
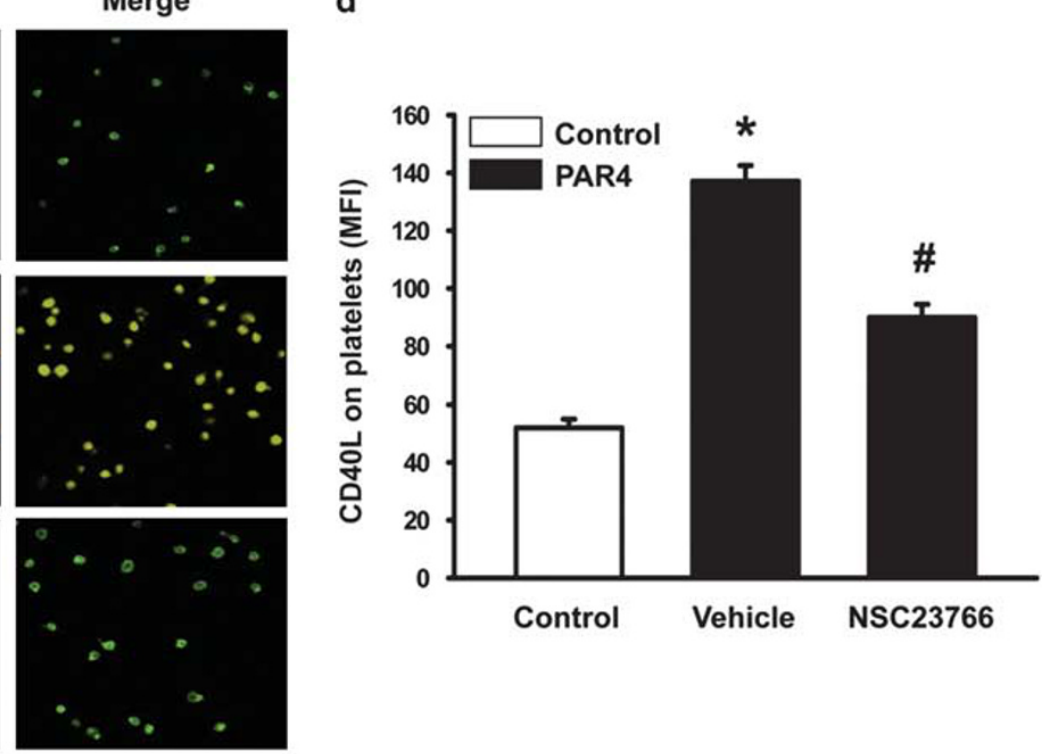

e

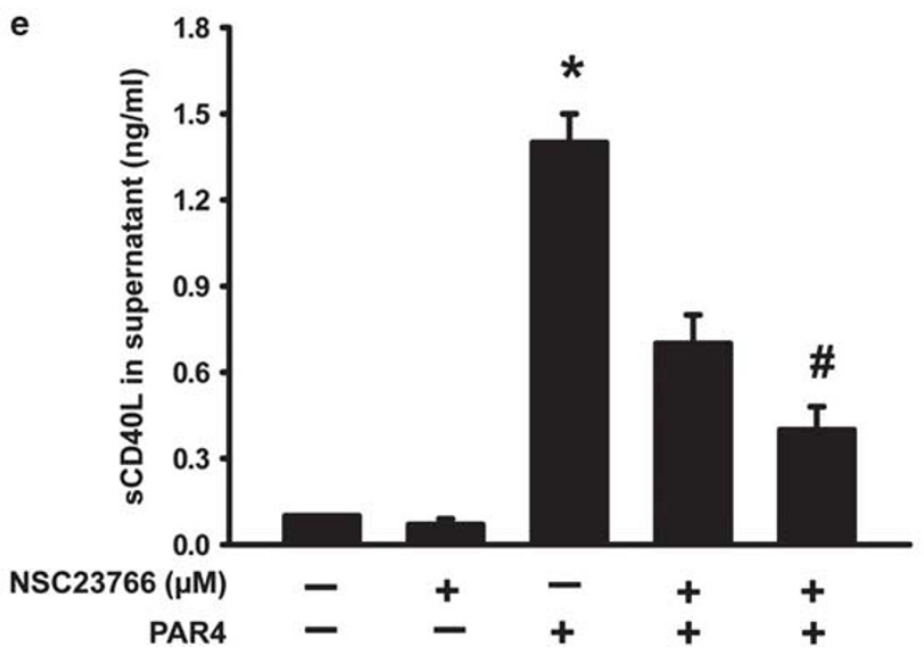


secretion from neutrophils. Immunostaining revealed abundant levels of MMP-9 in resting neutrophils (Figures $5 \mathrm{a}$ and b). Challenge with CXCL2 rapidly mobilized and decreased intracellular levels of MMP-9 in neutrophils (Figures 5a and b). Notably, preincubation with NSC23766 completely inhibited MMP-9 secretion and maintained normal levels of MMP-9 in neutrophils exposed to CXCL2 (Figures 5a and $\mathrm{b}$ ).

a

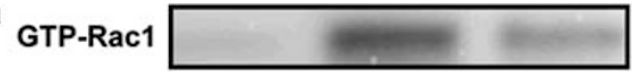

Total Rac1
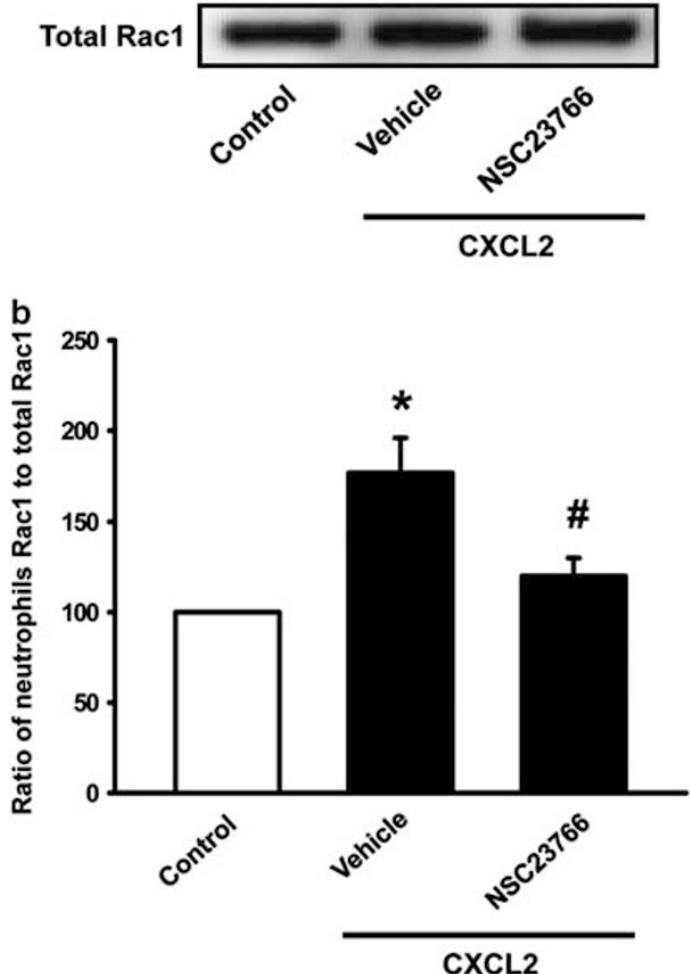

Figure 3 CXCL2 induces Rac1 activity in neutrophils. (a) Neutrophils were isolated by magnetic beads and then $1 \times 10^{6}$ neutrophils were incubated with CXCL2 $(0.3 \mu \mathrm{g} / \mathrm{ml})$ for $20 \mathrm{~min}$ with and without Rac1 inhibitor NSC23766 (N6-[2-[[4-(diethylamino)-1-methylbutyl] amino]-6methyl-4-pyrimidinyl]-2 methyl-4, 6-quinolinediamine trihydrochloride; $10 \mu \mathrm{M})$. Active Rac1 protein was pulled down from neutrophil lysates by using GST-PAK beads. Rac1-GTP was detected by western blot. (b) Band intensities were quantified by densitometry and normalized to total Rac1. Bars represent mean \pm s.e.m. and $n=4$. ${ }^{*} P<0.05$ vs control and ${ }^{\#} P<0.05$ vs vehicle + CXCL2.

\section{Neutrophil-Derived MMP-9 Regulates Platelet Shedding of CD40L}

We used MMP-9 gene-deficient mice to determine the role of neutrophil-derived MMP-9 in regulating platelet shedding of CD40L. Stimulation with PAR4 was used to mobilize CD40L on the surface of MMP-9-deficient platelets. It was found that supernatant from wild-type neutrophils stimulated with CXCL2 increased the shedding of surface expressed CD40L from MMP-9-deficient platelets from $0.02 \pm 0.0$ up to $1.4 \pm$ $0.08 \mathrm{ng} / \mathrm{ml}$, corresponding to a 53 -fold increase compared with control (Figure 6). Incubation of MMP-9-deficient platelets with supernatants from MMP-9 gene-deficient neutrophils stimulated with CXCL2 caused a significantly lower level of CD40L shedding (Figure 6). Stimulation with PAR4 significantly enhanced shedding of CD40L from MMP-9-deficient platelets by 28 -fold compared with control (Figure 6).

\section{DISCUSSION}

This study demonstrates that sepsis-induced platelet shedding of CD40L is dependent on Racl signaling. We found that Racl might regulate sepsis-induced plasma levels of CD40L at two distinct levels, that is, on one hand Rac1 controls surface mobilization of CD40L on activated platelets and on the other hand Rac1 regulates MMP-9 secretion from neutrophils. Taken together, our data suggest that Racl signaling coordinates multiple events leading to increased plasma levels of soluble CD40L in abdominal sepsis.

Accumulating data suggest that platelets exert multiple effects beyond promoting hemostasis such as proinflammatory actions. For example, it has been reported that platelets have a key role in polymicrobial sepsis by potentiating neutrophil recruitment to the lung in a contact-independent manner. ${ }^{30}$ Platelets contain numerous proinflammatory substances including chemokines and cytokines. ${ }^{31,32} \mathrm{We}$ have demonstrated that platelet-dependent pulmonary infiltration of neutrophils is mediated by soluble CD40L released from platelets in abdominal sepsis. ${ }^{8}$ In support of a role of CD40L in sepsis, elevated plasma levels of soluble CD40L have been observed in patients with sepsis. ${ }^{33,34}$ However, the specific signaling mechanisms regulating platelet shedding of CD40L in sepsis are not known. In the present study, we found that Racl activity was increased in platelets from septic mice. We therefore asked whether Rac1 activity might regulate platelet shedding of CD40L. It was found that platelet depletion abolished the sepsis-induced

Figure 2 Rac1 regulates platelet shedding of CD40L. (a) Plasma level of soluble CD40L (sCD40L) from sham and cecal ligation and puncture (CLP (6 h) animals. (b) A representative plot of flow cytometry showing the platelet depletion. (c) Representative image of confocal microscopy showing surface expression of CD40L on isolated platelets. (d) Summarized data showing mean fluorescence intensity (MFI) of CD40L expression on platelets. (e) Isolated platelets were activated with proteinase-activated receptor-4 (PAR4) (200 $\mu$ M) for 20 min with and without Rac1 inhibitor NSC23766 (N6-[2-[[4-(diethylamino)-1-methylbutyl] amino]-6-methyl-4-pyrimidinyl]-2 methyl-4, 6-quinolinediamine trihydrochloride; 10 and $100 \mu$ M), and then levels of soluble CD40L were determined in the supernatants by enzyme-linked immunosorbent assay. Nonstimulated platelets served as control. Bars represent mean \pm s.e.m. and $n=4-5$. ${ }^{*} P<0.05$ vs sham or control, ${ }^{*} P<0.05$ vs vehicle + CLP or vehicle + PAR4, and ${ }^{\text {a }} P<0.05$ vs Ctrl $a b+C L P$. 

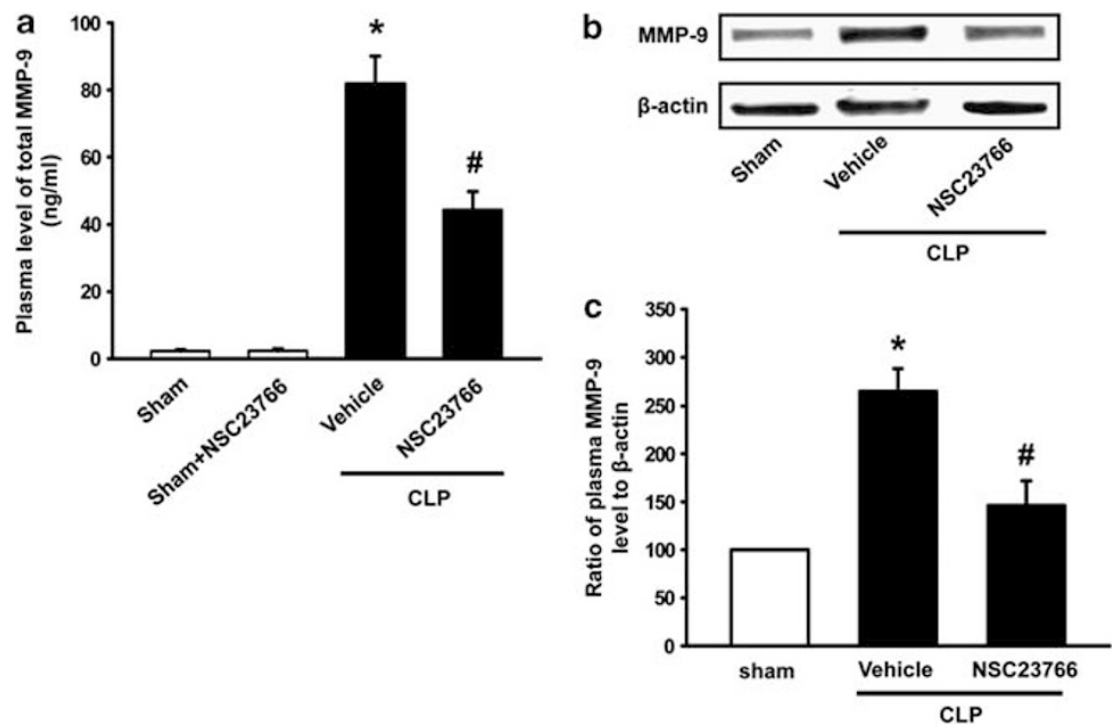

Figure 4 Plasma levels of matrix metalloproteinase-9 (MMP-9) are elevated in cecal ligation and puncture (CLP) mice. (a) Plasma levels of total MMP-9 $6 \mathrm{~h}$ after CLP. Sham-operated animals served as negative controls. (b) Plasma from sham and $6 \mathrm{~h}$ CLP mice were analyzed by western blot for the presence of MMP-9. (c) Band intensities were quantified by densitometry and ratio of plasma MMP-9 to $\beta$-actin is shown. Western blots are representative of four independent experiments. Bars represent mean \pm s.e.m. and $n=4$. ${ }^{*} P<0.05$ vs sham and ${ }^{\#} P<0.05$ vs vehicle + CLP.

a
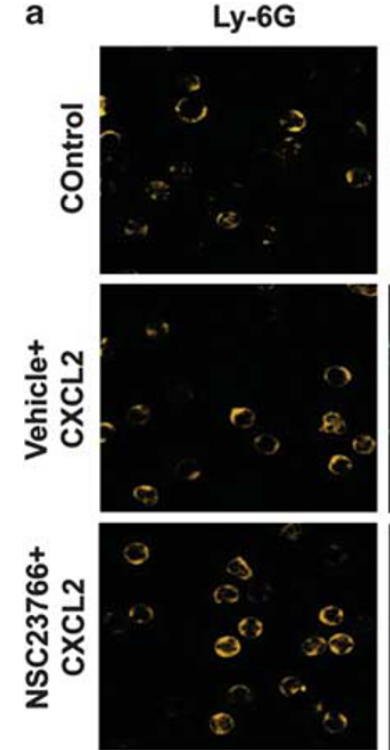

MMP-9
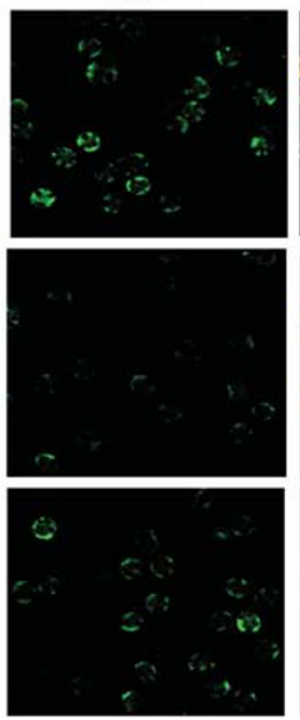

Merge
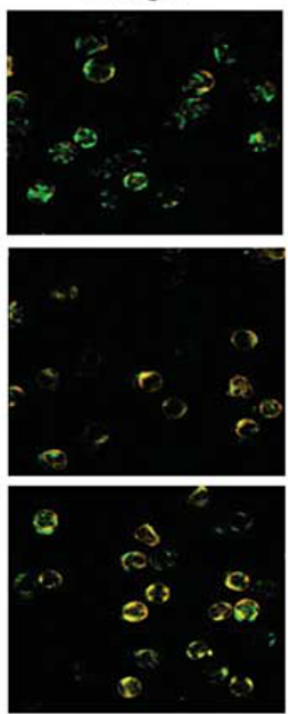

b

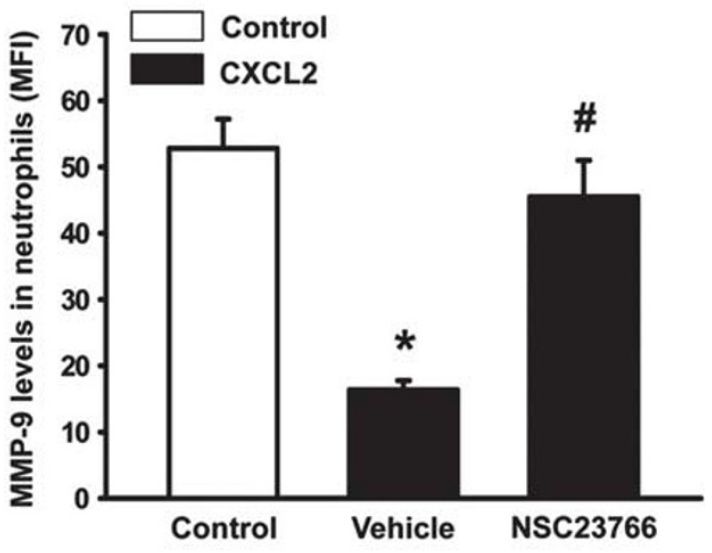

Figure 5 Rac1 regulates neutrophil secretion of matrix metalloproteinase-9 (MMP-9). (a) Neutrophils were isolated by magnetic beads. (a) Isolated neutrophils were incubated with CXCL2 $(0.3 \mu \mathrm{g} / \mathrm{ml})$ and then was the level of MMP-9 in permeabilized neutrophils determined by confocal microscopy. (b) Summarized data showing mean fluorescence intensity (MFI) of MMP-9 in neutrophils. Bars represent mean \pm s.e.m. and $n=4$. ${ }^{*} P<0.05$ vs control and ${ }^{\#} P<0.05$ vs vehicle + CXCL2.

increase of $\mathrm{CD} 40 \mathrm{~L}$ levels in the plasma, suggesting that platelets are the dominating source of soluble CD40L in sepsis, which is in line with our previous findings. ${ }^{10}$ Interestingly, we also found that NSC23766 completely inhibited the CLP-provoked enhancement of plasma levels of CD40L, indicating that Racl is an important regulator of platelet shedding of CD40L in abdominal sepsis. More- over, NSC23766 also abolished PAR4-induced surface expression and shedding of $\mathrm{CD} 40 \mathrm{~L}$ in isolated platelets in vitro, supporting the notion that Rac1 controls CD40L secretion from platelets. In this context, it is interesting to note that NSC23766 was recently shown to inhibit agonistinduced mobilization of P-selectin in platelets. ${ }^{19}$ Knowing that both $\mathrm{CD} 40 \mathrm{~L}$ and P-selectin are localized in the 


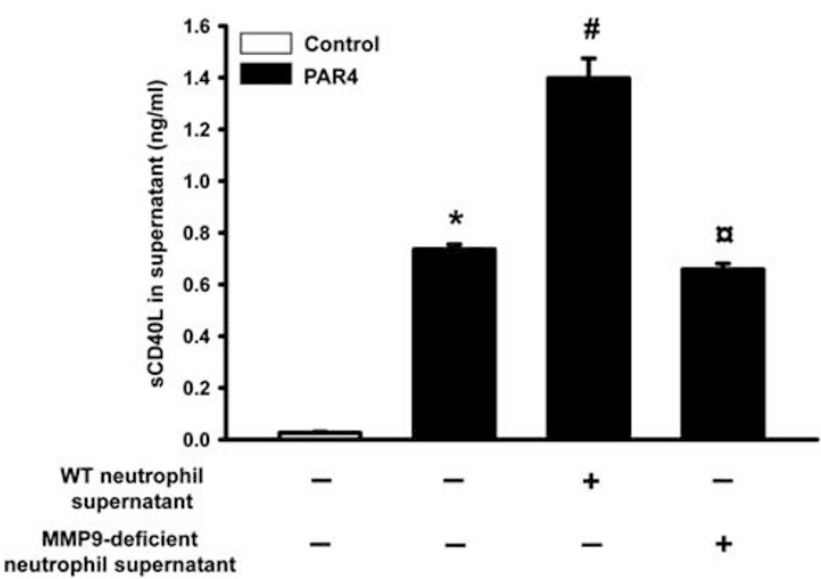

Figure 6 Neutrophil-derived matrix metalloproteinase-9 (MMP-9) regulates platelet shedding of CD40L. Isolated MMP-9 gene-deficient platelets were incubated with proteinase-activated receptor-4 (PAR4) and with supernatants from either wild-type or MMP-9-deficient neutrophils stimulated with CXCL2 for $30 \mathrm{~min}$. Soluble CD40L was determined in the supernatants by enzyme-linked immunosorbent assay. Nonstimulated platelets served as control. Bars represent mean \pm s.e.m. and $n=4-5$. ${ }^{*} P<0.05$ vs control, ${ }^{\#} P<0.05$ vs control, and ${ }^{\mathrm{a}} P<0.05$ vs wild-type neutrophil supernatant.

membrane of $\alpha$-granules in platelets, these findings suggest that Racl might be involved in the secretion of $\alpha$-granules in platelets.

The MMP family of proteases is generally considered to regulate degradation and formation of the extracellular matrix, but overwhelming data in the literature also implicate MMPs in several features of inflammatory reactions by regulating cleavage and shedding of surface molecules. ${ }^{35,36} \mathrm{In}$ fact, several studies have indicated that MMP-9 might have an important role in sepsis. ${ }^{8,37}$ One mechanism by which MMP-9 regulate proinflammatory actions in sepsis appears to be related to platelet shedding of CD40L. ${ }^{10}$ Using both ELISA and western blot, we observed herein that plasma levels of MMP-9 were increased in abdominal sepsis. This finding is in accordance with other investigations showing enhanced MMP-9 levels in the circulation of sepsis and infectious disease models, ${ }^{37-39}$ as well as in patients with sepsis. ${ }^{10,40}$ Notably, it was observed that administration of NSC23766 significantly decreased the sepsis-induced increase of MMP-9 levels in plasma, indicating that Racl might be an important regulator of systemic levels of MMP-9 in abdominal sepsis. It should be mentioned that two studies have reported that MMP-2 might promote platelet release of CD40L in vitro, ${ }^{41,42}$ but levels of MMP-2 in the plasma are not elevated in abdominal sepsis. ${ }^{10}$ The potential presence of MMP-9 in platelets has been a controversial subject in the literature. Some authors have reported that platelets do not contain significant amount of MMP-9, ${ }^{43,44}$ whereas others have shown that platelets do contain MMP-9. ${ }^{45-47}$ Nonetheless, it is well accepted that neutrophils are a rich source of MMP-9.29,48 We therefore asked whether Racl might have a role in MMP-9 secretion from neutrophils. It was found that stimulation with the neutrophil chemoattractant CXCL2 caused a significant increase in Racl activity in neutrophils. Using confocal microscopy, we observed clearcut levels of MMP-9 in isolated neutrophils and that CXCL2 challenge decreased neutrophil content of MMP-9, suggesting that CXCL2 stimulates MMP9 secretion from neutrophils. Interestingly, we found that administration of NSC23766 completely inhibited CXCL2induced secretion of MMP-9 in neutrophils, indicating that Rac1 regulates neutrophil secretion of MMP-9. Moreover, we found that supernatant from wild-type neutrophils stimulated with CXCL2 caused significant shedding of CD40L from MMP-9-deficient platelets. In contrast, supernatant from MMP-9 gene-deficient neutrophils stimulated with CXCL2 triggered significantly less CD40L shedding from MMP-9-deficient platelets, indicating that neutrophilderived MMP-9 is a potent regulator of platelet shedding of CD40L. In this context, it is interesting to note that PAR4 alone caused significant shedding of CD40L from MMP-9-deficient platelets, suggesting that MMP-9independent mechanisms could also be involved in platelet shedding of CD40L.

It should be mentioned that our present findings are limited to mice and one must be careful when extrapolating findings in experimental sepsis to human sepsis, considering significant differences in age and comorbidity between mice and humans. In addition, Racl is an important protein in intracellular signaling ${ }^{49-51}$ and risks of potential side effects should be considered. Therefore, further studies in human materials on the role of Racl for MMP-9 secretion and platelet shedding of CD40L are needed.

Taken together, these findings indicate that Racl activity is increased in platelets and regulates platelet shedding of CD40L in polymicrobial sepsis. Moreover, these results suggest that Rac1 signaling controls circulating levels of MMP-9 in sepsis and that Rac1 regulate neutrophil secretion of MMP-9. Thus, our data indicate that inhibition of Rac1 signaling might be a useful target to control pathologic secretion and shedding of CD40L into the systemic circulation in abdominal sepsis.

\section{ACKNOWLEDGMENTS}

This work was supported by grants from the Swedish Medical Research Council (2012-3685), Crafoord foundation, Einar and Inga Nilssons

foundation, Harald and Greta Jaenssons foundation, Greta and Johan Kocks foundation, Agnes Nilssons foundation, Franke and Margareta Bergqvists foundation for promotion of cancer research, Magnus Bergvalls foundation, Mossfelts foundation, Nanna Svartz foundation, Ruth and Richard Julins foundation, Swedish Medical Society fund, Malmö General Hospital foundation for combating cancer, MAS fund, and Skåne University Hospital and Lund University. RH is supported by Hawler Medical University and Kurdistan Regional Government. EZ's position receives funding from the EU Integrated project BetaBat and a collaborative SRC grant, as well as the Swedish Strategic Research EXODIAB. This work was carried out using imaging equipment financed by the Knut and Alice Wallenberg foundation. 


\section{AUTHOR CONTRIBUTIONS}

$\mathrm{RH}$ and MR performed experiments, analyzed data, and wrote the manuscript. EZ performed experiments and wrote the manuscript. HT supervised the project, designed the experiments, and wrote the manuscript.

\section{DISCLOSURE/CONFLICT OF INTEREST}

The authors declare no conflict of interest.

1. Cohen J. The immunopathogenesis of sepsis. Nature 2002;420: 885-891.

2. Heyland DK, Hopman $\mathrm{W}, \mathrm{Coo} \mathrm{H}$, et al. Long-term health-related quality of life in survivors of sepsis. Short Form 36: a valid and reliable measure of health-related quality of life. Crit Care Med 2000;28: 3599-3605.

3. Martin GS, Mannino DM, Eaton S, et al. The epidemiology of sepsis in the United States from 1979 through 2000. N Engl J Med 2003; 348:1546-1554

4. Asaduzzaman M, Zhang S, Lavasani S, et al. LFA-1 and Mac-1 mediate pulmonary recruitment of neutrophils and tissue damage in abdominal sepsis. Shock 2008;30:254-259.

5. Bacon KB, Oppenheim JJ. Chemokines in disease models and pathogenesis. Cytok Growth Factor Rev 1998;9:167-173.

6. Hasan Z, Palani K, Rahman M, et al. Targeting CD44 expressed on neutrophils inhibits lung damage in abdominal sepsis. Shock 2011, 35:567-572.

7. Zhang S, Song L, Wang Y, et al. Targeting CD162 protects against streptococcal M1 protein-evoked neutrophil recruitment and lung injury. Am J Physiol 2013;305:L756-L763.

8. Rahman M, Zhang S, Chew M, et al. Platelet-derived CD40L (CD154) mediates neutrophil upregulation of Mac-1 and recruitment in septic lung injury. Ann Surg 2009;250:783-790.

9. Zarbock A, Polanowska-Grabowska RK, Ley K. Platelet-neutrophil interactions: linking hemostasis and inflammation. Blood Rev 2007; 21:99-111.

10. Rahman M, Zhang $S$, Chew $M$, et al. Platelet shedding of CD40L is regulated by matrix metalloproteinase- 9 in abdominal sepsis. J Thromb Haemostas 2013;11:1385-1398.

11. Fan $\mathrm{H}$, Cook JA. Molecular mechanisms of endotoxin tolerance. $J$ Endotox Res 2004;10:71-84.

12. Kim JM, Oh YK, Lee JH, et al. Induction of proinflammatory mediators requires activation of the TRAF, NIK, IKK and NF-kappaB signal transduction pathway in astrocytes infected with Escherichia coli. Clin Exp Immunol 2005;140:450-460.

13. Itoh K, Yoshioka K, Akedo H, et al. An essential part for Rho-associated kinase in the transcellular invasion of tumor cells. Nat Med 1999; 5:221-225.

14. Loirand G, Guerin P, Pacaud P. Rho kinases in cardiovascular physiology and pathophysiology. Circ Res 2006;98:322-334.

15. Narumiya $\mathrm{S}$. The small GTPase Rho: cellular functions and signal transduction. J Biochem 1996:120:215-228.

16. Slotta JE, Braun OO, Menger MD, et al. Fasudil, a Rho-kinase inhibitor, inhibits leukocyte adhesion in inflamed large blood vessels in vivo. Inflam Res 2006;55:364-367.

17. Hwaiz R, Hasan Z, Rahman M, et al. Rac1 signaling regulates sepsisinduced pathologic inflammation in the lung via attenuation of Mac-1 expression and CXC chemokine formation. J Surg Res 2013;183: 798-807.

18. Etienne-Manneville $\mathrm{S}$, Hall A. Rho GTPases in cell biology. Nature 2002;420:629-635.

19. Akbar H, Kim J, Funk K, et al. Genetic and pharmacologic evidence that Rac1 GTPase is involved in regulation of platelet secretion and aggregation. J Thromb Haemostas 2007;5:1747-1755.

20. Flevaris $\mathrm{P}, \mathrm{Li} Z$, Zhang G, et al. Two distinct roles of mitogen-activated protein kinases in platelets and a novel Rac1-MAPK-dependent integrin outside-in retractile signaling pathway. Blood 2009;113: 893-901.

21. Guidetti GF, Bernardi B, Consonni A, et al. Integrin alpha2beta1 induces phosphorylation-dependent and phosphorylation-independent activation of phospholipase Cgamma2 in platelets: role of Src kinase and Rac GTPase. J Thromb Haemostas 2009;7:1200-1206.
22. McCarty OJ, Larson MK, Auger JM, et al. Rac1 is essential for platelet lamellipodia formation and aggregate stability under flow. J Biol Chem 2005;280:39474-39484.

23. Pandey D, Goyal P, Dwivedi S, et al. Unraveling a novel Rac1-mediated signaling pathway that regulates cofilin dephosphorylation and secretion in thrombin-stimulated platelets. Blood 2009;114:415-424.

24. Pleines I, Elvers M, Strehl A, et al. Rac1 is essential for phospholipase C-gamma2 activation in platelets. Pflug Archiv 2009;457:1173-1185.

25. Binker MG, Binker-Cosen AA, Gaisano HY, et al. Inhibition of Rac1 decreases the severity of pancreatitis and pancreatitis-associated lung injury in mice. Exp Physiol 2008;93:1091-1103.

26. Harada N, limuro $Y$, Nitta $T$, et al. Inactivation of the small GTPase Rac1 protects the liver from ischemia/reperfusion injury in the rat. Surgery 2003;134:480-491.

27. Yao HY, Chen L, Xu C, et al. Inhibition of Rac activity alleviates lipopolysaccharide-induced acute pulmonary injury in mice. Biochim Biophys Acta 2011;1810:666-674.

28. Rittirsch D, Huber-Lang MS, Flierl MA, et al. Immunodesign of experimental sepsis by cecal ligation and puncture. Nat Protocol 2009;4: 31-36.

29. Pugin J, Widmer MC, Kossodo S, et al. Human neutrophils secrete gelatinase $\mathrm{B}$ in vitro and in vivo in response to endotoxin and proinflammatory mediators. Am J Resp Cell Mol Biol 1999;20: 458-464.

30. Asaduzzaman M, Rahman M, Jeppsson B, et al. P-selectin glycoproteinligand-1 regulates pulmonary recruitment of neutrophils in a plateletindependent manner in abdominal sepsis. Br J Pharmacol 2009;156: 307-315.

31. Flad HD, Brandt E. Platelet-derived chemokines: pathophysiology and therapeutic aspects. Cell Mol Life Sci 2010;67:2363-2386

32. Yan Z, Zhang J, Holt JC, et al. Structural requirements of platelet chemokines for neutrophil activation. Blood 1994;84:2329-2339.

33. Chew $M$, Rahman $M$, Ihrman $L$, et al. Soluble CD40L (CD154) is increased in patients with shock. Inflam Res 2010;59:979-982.

34. Inwald DP, Faust SN, Lister $\mathrm{P}$, et al. Platelet and soluble CD40L in meningococcal sepsis. Intens Care Med 2006;32:1432-1437.

35. Ra HJ, Parks WC. Control of matrix metalloproteinase catalytic activity. Matrix Biol 2007;26:587-596.

36. Stamenkovic I. Extracellular matrix remodelling: the role of matrix metalloproteinases. J Pathol 2003;200:448-464.

37. Paemen $L$, Jansen PM, Proost $P$, et al. Induction of gelatinase $B$ and MCP-2 in baboons during sublethal and lethal bacteraemia. Cytokine 1997;9:412-415.

38. Teng L, Yu M, Li JM, et al. Matrix metalloproteinase-9 as new biomarkers of severity in multiple organ dysfunction syndrome caused by trauma and infection. Mol Cell Biochem 2012;360:271-277.

39. Torrence $A E$, Brabb $T$, Viney $J \mathrm{~L}$, et al. Serum biomarkers in a mouse model of bacterial-induced inflammatory bowel disease. Inflam Bowel Dis 2008;14:480-490.

40. Nakamura T, Ebihara I, Shimada $\mathrm{N}$, et al. Modulation of plasma metalloproteinase-9 concentrations and peripheral blood monocyte mRNA levels in patients with septic shock: effect of fiber-immobilized polymyxin B treatment. Am J Med Sci 1998;316:355-360.

41. Choi WS, Jeon OH, Kim DS. CD40 ligand shedding is regulated by interaction between matrix metalloproteinase- 2 and platelet integrin alpha(IIb)beta(3). J Thromb Haemostas 2010;8:1364-1371.

42. Reinboldt $\mathrm{S}$, Wenzel $\mathrm{F}$, Rauch $\mathrm{BH}$, et al. Preliminary evidence for a matrix metalloproteinase-2 (MMP-2)-dependent shedding of soluble CD40 ligand (sCD40L) from activated platelets. Platelets 2009;20: 441-444.

43. Cecchetti $\mathrm{L}$, Tolley ND, Michetti N, et al. Megakaryocytes differentially sort mRNAs for matrix metalloproteinases and their inhibitors into platelets: a mechanism for regulating synthetic events. Blood 2011; 118:1903-1911.

44. Galt SW, Lindemann S, Medd D, et al. Differential regulation of matrix metalloproteinase- 9 by monocytes adherent to collagen and platelets. Circ Res 2001;89:509-516.

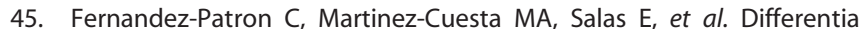
regulation of platelet aggregation by matrix metalloproteinases- 9 and -2. Thromb Haemostas 1999:82:1730-1735.

46. Mannello F. Serum or plasma samples? The 'Cinderella' role of blood collection procedures: preanalytical methodological issues influence the release and activity of circulating matrix metallo- 
proteinases and their tissue inhibitors, hampering diagnostic trueness and leading to misinterpretation. Arterioscler Thromb Vasc Biol 2008; 28:611-614.

47. Menchen L, Marin-Jimenez I, Arias-Salgado EG, et al. Matrix metalloproteinase 9 is involved in Crohn's disease-associated platelet hyperactivation through the release of soluble CD40 ligand. Gut 2009;58:920-928.

48. Lindsey M, Wedin K, Brown MD, et al. Matrix-dependent mechanism of neutrophil-mediated release and activation of matrix metallo- proteinase 9 in myocardial ischemia/reperfusion. Circulation 2001; 103:2181-2187.

49. Katsumi $A$, Milanini J, Kiosses WB, et al. Effects of cell tension on the small GTPase Rac. J Cell Biol 2002;158:153-164.

50. Razzouk S, Lieberherr M, Cournot G. Rac-GTPase, osteoclast cytoskeleton and bone resorption. Eur J Cell biol 1999;78:249-255.

51. Woo CH, Jeong DT, Yoon SB, et al. Eotaxin induces migration of RBL$2 \mathrm{H} 3$ mast cells via a Rac-ERK-dependent pathway. Biochem Biophys Res Commun 2002;298:392-397. 